\begin{tabular}{|c|c|}
\hline $\begin{array}{l}\text { Journal of } \\
\text { Radiotherapy } \\
\text { in Practice }\end{array}$ & $\begin{array}{l}\text { Yournal of Radiotherapy in Practice (2008) } \\
\text { 7, } 185-194 \\
\text { ○ } 2008 \text { Cambridge University Press } \\
\text { doi:10 1017/S14603969080060707 }\end{array}$ \\
\hline
\end{tabular}

\title{
Abstracts
}

\section{Abstracts from papers presented at the Radiotherapy in Practice 4 Conference, Sheffield Hallam University, UK}

\section{0-12th October 2008}

\section{PARTICLE THERAPY \\ Juergen Salk \\ Paul-Scherrer-Institute, Switzerland. \\ E-mail: juergen.salk@psi.ch}

The use of protons for cancer treatment was first suggested by Robert R. Wilson in 1946. The major advantage of protons and other heavy charged particles is that their dose deposition varies almost inversely with the particle energy. Thus, the absorbed dose is low while the beam traverses normal tissues until nearly the end of the particle's range, where the dose deposit peaks out to a high value at the Bragg Peak. This is followed by a rapid falloff to zero dose beyond the distal end of the tumour.

The first patient treatments with protons were performed at the Lawrence Berkley Laboratories in 1954 and more than four decades this pioneering work has been continuously refined and expanded in a number of research facilities worldwide. The most traditional and still predominant approach for proton delivery is the passive scattering technique. This method depends on one or more scatter foils for lateral beam broadening and properly designed collimators and compensators to define the aperture and distal end shaping of the beam. The energy of the incident particles is modulated by means of a rotating range-shifter wheel with varying thickness in order to spread out the Bragg peak over the tumour volume dimensions along the beam direction.
A more advanced delivery technique uses dynamic beam scanning. This method does not require any scattering foils or patient specific hardware such as collimators and compensators. With active spot scanning, the position and relative weight of each individual Bragg peak can be chosen freely in three dimensions such that the dose can be precisely tailored to the three-dimensional shape of the target volume with significant reduction of dose on healthy tissue surrounding the tumour. The practical feasibility of this approach has been demonstrated clinically at the Paul-ScherrerInstitute (PSI) in Switzerland for more than 10 years with more than 300 patients treated. Spot scanning is now being recognised internationally as the preferred delivery technique for charged particles in general because of its potential for intensity modulated particle therapy (IMPT). In this talk we will present various clinical aspects of particle therapy as well as open questions and new challenges currently being worked on in this field.

\section{RADIATION THERAPISTS' \\ UNDERSTANDING AND \\ INTERPRETATIONS OF \\ CARING: CORE VALUE \\ OR VALUE-ADDED EXTRA?}

\section{Amanda Bolderston}

Princess Margaret Hospital, Toronto, Canada. E-mail: amanda.bolderston@rmp.uhn.on.ca 
The concept of caring has been extensively studied in the field of nursing and is generally understood to be one of nursing's core values. However, although therapists generally claim to care for their patients, there has been no attempt to quantify what caring means to the therapists themselves. In addition, nurses have experienced an increasing reliance on machinery and equipment, especially in areas such as critical care. This had led to claims that technological competence rather than humanistic caring now drives nursing in such areas. Although radiation therapy has always had a strong technological focus, the last decade has seen profound change and an unprecedented increase in automation and computerisation in patient treatment. The impact of this dramatic change on patient care in radiation therapy is unknown. This study thus explores radiation therapists' understanding and interpretations of caring within their profession. It attempts to discover whether therapists have a definition of caring unique to their highly technical profession and whether caring changes according to the individual's experience, access to sophisticated technology and techniques and other variables. A qualitative approach is used with focus group methodology. Comparative data analysis of the transcribed discussions is carried out using content analysis and categorised according to the emergent themes. It is hypothesised that the concept of care will be integral to the therapists' professional practice, although there will likely be elements that differ from the nursing literature.

\section{USER INTERFACE DESIGN FOR SAFETY IN RADIOTHERAPY}

\section{Teresa Clark}

\author{
Medical Physics Department, Cumberland Royal \\ Infirmary, Carlisle, UK. \\ E-mail:teresa.clark@nhs.net
}

Significant attention has been paid to improved user interface design for safety in infusion systems. Radiotherapy systems provide potentially greater risk of harm to patients, yet there is very little significant research into the design of the user's interaction with these complex systems.
A survey of the equipment available in a typical radiotherapy unit reveals numerous user interface design faults. Parallel design faults on infusion pumps have resulted in wholesale FDA recalls, examples are provided. Yet rates of adverse events in radiotherapy are low; a current systematic survey of the literature is used to show this. Fatality rates are significantly lower than those claimed for infusion therapy.

There is much concern over recent serious overexposures, and a proposal for systems review by the NPSA (National Patient Safety Agency). However, current practice in radiotherapy is founded on a solid basis of quality control and vigilance which appears largely effective in preventing accidents. Radiotherapy is administered in a carefully controlled environment, by highly trained staff. By contrast infusions are administered everywhere within the health care environment, under circumstances which often predispose towards distraction and therefore human error, and with no regulatory requirement for training.

Can radiotherapy safety be improved still further by user interface design for patient safety? Can standardisation, promoted by NPSA and others as part of the solution in infusion therapy, be applied to radiotherapy? Factors considered include contribution of poor design to past incidents, lack of standardisation, lack of adoption of standards, layout and prioritisation of information on displays, consistency of dosing units from prescription to administration, software based safety and checking systems, keypad layout and others. Opportunities for learning are proposed from the two models.

READABILITY AND LEGIBILITY OF PRINTED INFORMATION LEAFLETS IN RADIOTHERAPY

\section{David Flinton}

City University, London, UK.

E-mail:d.m.flinton@city.ac.uk

\section{Background}

The objective of this work was to undertake a descriptive study to assess the readability and 
legibility of Printed Information Leaflets (PILs) currently being used by radiotherapy departments in England and Wales.

\section{Method}

Twenty-five departments were requested to supply their patient information leaflets. Seventeen departments did so, giving a return rate of 68\%. All the patient information leaflets were then assessed for ease of readability using a number of readability formulas. Use of passive voice was assessed using three common word processing packages, and legibility was assessed by comparing documents to guidelines set out by the NHS toolkit for producing patient information (2003), using a tool specifically designed and tested for this purpose.

\section{Results and Conclusions}

The average readability level (13.7) was acceptable, though many PILs had a readability level that was too high for the majority of the population to understand. The use of passive voice in most PILs was too high making the document longer and harder to understand than necessary, and it is recommended that that both these issues should be addressed in future leaflets produced. Most leaflets scored highly for general presentation. There were, however, some areas of presentation that could be improved which in turn should aid legibility, specifically justification, explanation of instructions and use of images which did not comply with the NHS guidelines. Quality ratings on corporate identity were low.

\section{A GRADUATED SCENARIO: AN EXERCISE IN REFLECTIVE LEARNING}

\section{Jenny Moon}

Bournemouth University, UK.

E-mail:jmoon@bournemouth.ac.uk

The session will be devoted entirely to an exercise that is designed to support initial learning in reflective writing and the development of understanding of how to deepen and improve the quality of reflection. The exercise can be used directly for student groups as well as for teaching staff or in professional development. It is based on work with a story and on group discussion but is supported by a framework that can guide future reflective writing.

This and other similar exercises are free for use. Web addresses of resources will be indicated.

\section{UNDER GRADUATE REFLECTION- SUPPORTING THE JOURNEY \\ Monica Norris, Jane Harvey \\ University College Suffolk, Ipswich, UK. \\ E-mail:monica.norris@nnuh.nhs.uk}

At the University Campus Suffolk, evaluation of the practice placement portfolio for the BSc (Hons) Oncology and Radiotherapy Technology degree has initiated the development of the portfolio's reflective component for firstyear students. This is in response to students indicating difficulty in engaging in meaningful reflection and additionally takes cognisance of the HPC and local curriculum's requirement for the development of a reflective practitioner.

It is recognised that the value of a portfolio lies in students documenting and reflecting on experience during clinical placements, thereby enhancing learning and understanding. To develop this process, three essential criteria were identified for inclusion in a reflective tool: the consolidation of prior reflective knowledge, the opportunity to practice reflection safely with guidance and finally, an opportunity for students to discuss reflection thereby extending their insight into the reflective process. Further to this, privacy and confidentiality for students were considered crucial elements to encourage student participation.

An electronic learning tool has been devised to facilitate students working through a systematic process individually, culminating in the production of a reflective statement. This statement will form the basis of a clinical tutorial with the student's peer group and clinical lecturer, in the clinical setting. Course Team 
approval has been obtained to pilot this tool for first-year students during their second semester. This is an action research project and it is envisaged that evaluation of the pilot study will lead to the introduction of this tool as part of the degree programme from September 2008.

\section{ADDRESSING CPD FOR HPC/KSF IN A RADIOTHERAPY DEPARTMENT}

\section{Hema Patel, Emma Hallam}

Nottingham City Hospital, UK.

E-mail:Hema.Patel@nuh.nhs.uk

The Radiotherapy profession has seen many changes this past decade, Implementation of mandatory CPD, Agenda For change (AFC) and the Knowledge and Skills Framework (KSF).

\section{Aims}

The aim is to address how this department is going to assist staff to engage in CPD for HPC requirement and KSF.

\section{Objectives}

i. Familiarise all staff with KSF strategies and HPC requirements.

ii. Identify staff concerns regarding CPD/KSF.

iii. Revising the Nottingham CPD folder to include KSF objectives.

iv. Establishing a journal review club.

\section{Main issues of concern identified}

i. Finding the time to evidence practice.

ii. How to evidence the ongoing learning.

iii. Creating opportunities for CPD.

\section{Addressing the issues}

Time: Use of service days, CHART time and informal agreement with the Radiotherapy manager regarding working hours and CPD study time. Evidencing everyday PracticeRevision of the Nottingham CPD folder with template forms.

Creating opportunities for CPD: Setting up of a journal review club to get everyone involved and lunchtime talks.

\section{Summary}

To summarise, the concerns for evidencing practice for CPD and KSF have been addressed to a certain degree, whereby some agreement has been made with regards to CPD study time and the development of a folder to help get individuals started with evidencing. The matching of evidence to both CPD and KSF has alleviated some fears of double effort being made and finding time to evidence. The template forms, together with communicating how to evidence, have given staff a clearer image of what is expected of them.

\section{AN ACUPUNCTURE SERVICE IN ONCOLOGY \\ Jenny Salmon \\ Royal Cornwall Hospital, Truro, UK. \\ E-mail:jenny.salmon@rcht.cornwall.nhs.uk}

\section{Aim}

To conduct an 18-month study to evaluate the introduction of an acupuncture service for oncology patients in Cornwall.

\section{Methodology}

The study was an observational study of a case series. Patients were referred for one or more of the following: hot flushes, nausea, vomiting, pain, breathlessness, dry mouth, constipation, anxiety, depression, fatigue, relaxation, wellbeing and support. Patients received six or more sessions of acupuncture. Treatments were 20-30 minutes duration. Data were collected from participants on day 1 and at the final treatment session. This paper reports on the patient experience of the service with a particular focus on symptom grade.

\section{Results}

A total of 107 referrals were made. Data are available for 32 participants; 2 patients refused further treatment; 17 were too ill to complete their course of treatment; the remainder failed to attend their final appointment for unknown reasons and their data is incomplete. $62.5 \%$ of patients showed improvement in the grade of the symptom for which they were referred. 
9.4\% had no change in their symptom. $28.1 \%$ experienced deterioration in their symptom. The largest group of participants (21 complete data sets) were referred for hot flushes. 57.1\% showed improvement in symptom scores. $14.3 \%$ had no change in their symptom. $28.5 \%$ experienced deterioration in their hot flushes.

\section{Conclusion}

The results of this study suggest that an acupuncture service for Oncology is practicable and that a future acupuncture trial with a sophisticated experimental design focussing on the use of acupuncture for hot flushes associated with hormonal treatments for cancer would be worthwhile.

\section{IDENTIFYING THE CURRENT AND FUTURE ROLES OF THERAPY RADIOGRAPHERS AND THEIR ASSOCIATED OPPORTUNITIES AND CHALLENGES FOR ROLE DEVELOPMENT}

\section{Alison Smith}

Rosemere Cancer Centre, Preston UK and Angela Cross, University of Liverpool, UK. E-mail: alison. smith@lthtr.nhs.uk

In the current health care climate, therapy radiographers recognise the significant changes to their work patterns, professional roles and levels of accountability. The introduction of a Knowledge and Skills Framework, aligned with The Radiographer's 4 Tier Structure, may support the developments of radiographer's skills, knowledge and competencies in line with these changes.

To date, anecdotal evidence demonstrates how therapeutic radiographers at the practitioner level execute their role. Variations in role definitions including advanced practice, role development and role extension have resulted in a diverse range of definitions of duties. Coupled with local demands and local interpretation of roles, practitioners may experience limited or enhanced opportunities to develop and advance.
The presentations describe a research study undertaken a regional cancer centre. Thirty practitioners consented to interview, data were collected on their day-to-day professional activities. This data also recognised the opportunities and challenges individuals reported for their role development.

The aim of this presentation is to portray the legitimate research 'journey' undertaken by the researchers, including ethical and resource considerations.

The presentation also aims to highlight the benefit of conducting research within the 'naturalist' context of professionals working life. This works demonstrated the complexity of research in the health care settings and its value in attempting to interpret the roles, needs and the aspirations of the future practitioner.

\section{A PHASE II STUDY TO DETERMINE THE EFFICACY OF ALOE VERA GEL IN THE MANAGEMENT OF SKIN REACTIONS IN PATIENTS RECEIVING CURATIVE RADIATION THERAPY FOR CANCERS OF THE HEAD AND NECK}

\section{Annette Sperduti}

Princess Margaret Hospital, Toronto, Canada. E-mail: annette.sperduti@rmp.uhn.on.ca

This was a phase II study to determine the efficacy of Aloe Vera Gel in the management of skin reactions in patients receiving radiation therapy for cancers of the head and neck area.

\section{Methods}

Patients were asked to apply Aloe Vera Gel to either the left or right side of the treated volume twice daily commencing on day one of treatment (after radiation), and continuing until 2 weeks after radiation treatment is completed. The contra lateral side received the centres' routine skin care. The skin reactions for the right and left sides were scored independently each week by two assessors using the RISRAS and RTOG assessment scales. Patients were also assessed using a patient symptom scale to 
record symptoms such as tenderness, burning and itching. To validate the assessor's scores, weekly readings of the skin tone were taken using a Minolta chromo meter ${ }^{\circledR}$.

\section{Results}

A total of 31 patients were enrolled in the study. Statistically significant differences were observed in the proportion of patients scoring the aloe side better/worse across all weeks for the burn score $(\mathrm{McNemar} p$-value $=0.022$ and adjusted $p$-value $=0.015)$ and for the dry desquamation score $(\mathrm{McNemar} p$-value $=0.027$ and adjusted $p$-value approached significance $=0.079$ ). The side with the aloe gel applied was scored worse on $9(7 \%)$ occasions for the burn score and on $16(13 \%)$ occasions for the dry desquamation score. On only 1 (1\%) occasion the side with aloe gel applied scored better in the burn symptom score, and on only 5 (4\%) occasions for the dry desquamation score.

\section{Conclusion}

There does not appear to be any statistically significant advantage to the use of the aloe gel. Conversely, there appears to be some slight evidence that patients score symptoms slightly worse more often on the side of their neck in which the aloe gel was applied.

\section{TRAINING NEEDS OF RADIOGRAPHERS ASSOCIATED WITH IGRT}

\section{Suzanne Stanley}

St Fames's University Hospital, Leeds, UK.

E-mail:suzanne.stanley@leedsth.nhs.uk

IGRT is becoming a common technology in departments in the UK using $\mathrm{kV}$ or MV methods based on the linac. Training for IGRT usually begins with manufacturer applications training where the user can become adept in the acquisition of the image and start to understand the principles of image registration. However, following this currently departments must develop their own training packages to ensure the safe and competent operation of the kit. In Leeds, our strategy was to use the expertise of a consultant clinical oncologist and physicist who had prior experience of Synergy combined with a consultant radiologist. Six radiographers trained 'on the job' and after 6 months we were operating a radiographer-led service. Since then training has been competency based using a case study approach. We have identified issues/questions with training including:

- the need for 3D anatomy training;

- time taken to become competent;

- the level of experience required to acquire the skills that are required for image registration and subsequent correction strategies;

- maintenance of skills particularly related to the rotation/exit of staff.

This presentation questions the value of the methods used and looks at whether there are better methods that can be employed to deal with the requirement for therapy radiographers to develop skills beyond those achieved during undergraduate training and the routine radiotherapy work environment.

\section{CONFORMAL PROSTATE \\ RADIOTHERAPY: HOW \\ HAS THE ROUTINE USE \\ OF CONE BEAM CT \\ INFLUENCED CLINICAL \\ PRACTICE?}

\section{Helen Summers}

St Fames's University Hospital, Leeds, UK.

E-mail:helen.summers@leedsth.nhs.uk

Aim

Analyse the impact of cone beam CT (CBCT) off-line imaging protocols in prostate radiotherapy. Identify factors to select at planning which patients may benefit from CBCT instead of or alongside MV portal imaging.

\section{Methodology}

Retrospective review of CBCT images for 32 patients receiving conformal radiotherapy (55 Gy: 20 daily fractions). Patients received information on diet; some received regular laxatives prior to treatment. CBCTs acquired off-line fractions 
1-3 and once weekly. Images were reviewed by clinician and radiographer. Bone and CTV set up errors (SUEs) recorded and population averages calculated. AP rectal diameters measured at superior, isocentre and inferior planes. Interventions: isocentre shifts (if systematic CTV SUEs $>5 \mathrm{~mm}$ ), further diet advice or laxative (Fybogel or Movicol). Intervention rate was assessed.

\section{Results}

Mean CTV population SUEs $<3 \mathrm{~mm}$ in all directions. CTV SUE exceeded bone SUE for $59 \%$ of patients. AP displacements accounted for $90 \%$ of CTV SUEs. $75 \%$ of imaged fractions resulted in intervention compared to $1 \%$ if bony SUEs had been used alone. Population mean AP rectal diameter at isocentre $=4.2 \mathrm{~cm}$. Larger rectal diameter not directly proportional to larger CTV SUE, but differences in diameter $>1 \mathrm{~cm}$ between superior, isocentre and inferior levels associated with greater CTV errors. Saggital rectal shapes at CT planning categorised: Pyramid, Inverse Pyramid, Diamond, Hourglass and Regular. Inverse pyramid and diamond associated with greater CTV SUEs.

\section{Conclusions}

Workload increased with CBCT compared to standard 2-D approaches but did allow more accurate treatment delivery. Patients who may benefit from CBCT identified at planning by size and saggital rectal shape.

\section{PROSPECTIVE COHORT SURVEY OF PATIENT SATISFACTION WITH ON- TREATMENT REVIEW BY AN ADVANCED PRACTICE UROLOGY RADIOGRAPHER}

Fo Treeby

Addenbrookes Hospital, Cambridge, UK. E-mail:jo.treeby@addenbrookes.nhs.uk

Radiographers are undertaking roles that are a development and extension of what has traditionally been seen as their remit. As with any development it is important that such changes in practice have a positive rather than negative impact on patient care and the patient experience. This paper looks at the patient perception and level of patient satisfaction having 'on treatment review' conducted by a site specific advanced practitioner radiographer rather than an oncology doctor. Overall results were very positive suggesting that such reviews, when conducted by an appropriately trained individual, can enhance patient care and provide a satisfactory level of support during treatment.

\section{PEER SUPPORT GROUPS FOR THERAPY RADIOGRAPHERS}

\section{Joyce Butters, Jane Mathlin}

Velindre Cancer Centre, Cardiff, UK. E-mail:Foyce.butters@velindre-tr.wales.nhs.uk

The authors were concerned that their roles as specialist radiographers in the radiotherapy department could lead to them feeling isolated in their practice. Therapy radiographers are used to working in a small team on a linear accelerator. Role development has meant that this is no longer the case for the specialist radiographer.

The aim was to enable a group of therapeutic radiographers to gain support from each other in a safe environment.

A group of six radiographers meet once a month for 1 hour to discuss issues relating to practice. At the first meeting the ground rules were agreed and boundaries set. The group facilitate their own meetings.

These meetings have been running for just over a year. Meetings are held at the same time each month. Not all members have been able to attend all meetings but lively discussion has always ensued.

Group members have reported to have found the meeting helpful using the following words to describe the experience: safe, stress relief, reassuring, supportive, honest and de-isolating. 
Examples of issues discussed include the following:

- Grief issues after a patient has died

- Getting involved with patients beyond our clinical remit

- Dealing with other professional's behaviour

- Learning from sharing experiences

- Becoming more reflective.

Every participant has felt a benefit from the meetings. Reflecting on our practice has meant we are more effective in our roles and therefore provide a better service for patients and set a good example for colleagues. These meetings are now extended to other members of staff with the original radiographers leading these groups.

\section{EFFECTIVE USE OF POSITIONAL DATA THROUGH A SIMPLE AUDIT TOOL}

J. Stratford ${ }^{1}$, S. Smith ${ }^{2}$, H. Pennington ${ }^{1}$, M. Duffy ${ }^{1}$, J. Davies ${ }^{2}$, R. MacKay ${ }^{2}$

${ }^{1}$ Wade Centre for Radiotherapy Research, Christie Hospital, NHS Foundation Trust, UK, ${ }^{2}$ North West Medical Physics, Christie Hospital, Manchester, UK.

E-mail:Fulie.Stratford@christie.nhs.uk

\section{Introduction}

Responding to RCR recommendations ${ }^{1}$ regarding positioning variance, this institution has introduced a system to audit measured uncertainties in patient set up facilitating evaluation of random and systematic variations. Originally positional statistics were manually input into spreadsheets for analysis. This proved time consuming and open to manual transcription error. A relational database has been implemented that automatically gathers information from the Pinnacle planning system, Synergy verification system and IMPAC patient management system.

\section{Methods}

The database is a versatile system providing individual electronic patient records showing displacements, systematic/random trends, corrections and actions undertaken. Reports generated can identify trends for any required subgroup over set time frames. This is essential in the introduction of new techniques, immobilisation and technology. The system permits alternate margin recipes to be applied to the data.

\section{Results}

Currently more than 500 patient data sets have been collected and reports generated. Initial analysis concentrated on the evaluation of set up displacements for thoracic patients only and has identified several issues including problems with the existing immobilisation and the current correction strategy used for this group. It has provided valuable information on the effectiveness of current set up margins.

\section{Conclusion}

The relational database provides a powerful tool to effectively utilise the data collected. This is essential for image-guided radiotherapy to have a significant impact in our practice and ensure practice is evidence based. This presentation will describe the design and functionality of the database as well as examine the reports generated and how this has ultimately led to a change in practice.

\section{References}

1. The Royal College of Radiologists. Development and implementation of conformal radiotherapy in the United Kingdom. London: The Royal College of Radiologists; 2002.

\section{DELIVERING AN EFFECTIVE IGRT SERVICE WITH LIMITED RESOURCES}

\author{
J. Davies ${ }^{1}$, J. Stratford ${ }^{1}$, M. Duffy ${ }^{1}$, \\ H. Pennington ${ }^{1}$, C. Rowbottom ${ }^{2}$, \\ P. Whitehurst ${ }^{2}$ \\ ${ }^{1}$ Wade Centre for Radiotherapy Research Christie \\ Hospital NHS Foundation Trust, UK, \\ ${ }^{2}$ North Western Medical Physics Christie Hospital, \\ NHS Foundation Trust, Manchester, UK.
}

\section{Introduction}

Three-dimensional image guided radiotherapy has been established at this institution since 
2003. During this time the concept and benefits of this form of verification has become established with demand far exceeding supply. This technology has become a driving force for the future of radiotherapy and is reinforced by the recent recommendations made by the National Radiotherapy Advisory Group (NRAG), ${ }^{1}$ and The Cancer Strategy. ${ }^{2}$

\section{Discussion}

Following the successful implementation of IGRT into clinical practice, it became evident the gold standard would offer all patients a comprehensive 3D IGRT programme which leads to adaptive radiotherapy based on internal anatomy changes. Limited resources however, have resulted in a selective and reactive approach to treatment, with established criteria for patient selection and treatment complexity. This system of work ensures that patients receiving 3D IGRT receive the optimum standard of care and intervention where required. The author recognises these criteria would change on a departmental basis dependent on priorities and restrictions.

This strategy has resulted in role expansion for radiographers undertaking IGRT not only in terms of analysis but recognising the need for intervention and the impact this has on other resources and departments.

Training and education has a major role to play; establishing and maintaining competency is critical. The extended role of the radiographer may result in higher morale and greater job satisfaction; however in the current climate, this may not increase staff retention as with increased knowledge and expertise radiographers become a commodity.,

\section{References}

1. Radiotherapy: developing a world class service for England, Report to Ministers from National Radiotherapy Advisory Group 26 February 2007.

2. The Cancer Reform Strategy, Department of Health, December 2007.
3. Department of Health. Skills mix: A report on the fourtier service delivery model. London: Stationery Officer, 2003.

4. Hackman and Oldham. 'Motivation through Design at Work' Journal of Management. Risk Publication's.

\section{PARTICLE THERAPY REFERRALS TO EUROPE}

\section{Donna Routsis}

Addenbrookes Hospital, Cambridge, UK.

E-mail:donna.routsis@addenbrookes.nhs.uk

In April 2006, the National Radiotherapy Advisory Group (NRAG) proton subgroup submitted their advice for the future of proton services in the UK. Recommendations were made to establish a proton centre in the UK, providing the equality of access to advanced treatments as our European counterparts.

Interim recommendations were also made to create referral mechanisms for UK patients to access proton therapy aboard. Approval was granted in September 2007 and a working party was established to work with the National Commissioning Group (NCG) to create the pathways and processes needed.

The Proton Clinical Reference Panel (CRP) is a virtual panel of UK experts from many medical disciplines that advise the NCG, determines standards for treatment abroad and approves individual referrals, while individual patient management remains with the local referrer. Patients therefore have access to health professionals with proton expertise and referrals for treatments abroad are to centres providing high quality standards of treatment and care. This mechanism resolves both the current problem of inequitable referrals for patients and the funding and logistics issues for commissioners and Primary Care Trusts (PCTs).

Additional benefits are that as particle therapy becomes a more widely available treatment option, health care professionals gain the knowledge and skills necessary for the future establishment of a UK particle therapy facility. 
From April 2008, UK patients have been able to be assessed, referred and funded for quality proton treatments aboard. This talk will outline the processes and mechanisms involved.

\section{COMPARING ATTITUDES OF \\ PATIENTS, HEALTH \\ PROFESSIONALS AND \\ THE PUBLIC TOWARDS \\ RADIOTHERAPY REGIMES: \\ A SYSTEMATIC REVIEW}

\section{Heidi Probst}

Sheffield Hallam University, Sheffield, UK.

E-mail: H.Probst@shu.ac.uk

\section{Background}

To allow patients greater opportunity to be involved in the decision-making process it is necessary to understand how and when information should be provided, and considerable research has been undertaken to help practitioners in this respect. ${ }^{1-3}$ But how much do we know about patients' acceptance of radiotherapy regimens where there may be significant morbidity with varying benefit in terms of either survival or local control? And does level of acceptance of these regimens vary between patients and cancer professionals?

This review aims to identify current evidence pertaining to any mismatch between patient, public and health care workers perspectives of the side effects deemed acceptable for different levels of treatment outcome (i.e., percentage prolongation of life or local control) following radiotherapy treatment. The work is based on some seminal work in the field of chemotherapy by Slevin et al. ${ }^{4}$ We aimed to identify comparable research within radiation therapy to identify the need for a primary study. The work is important given the increasing emphasis on patient involvement in the decision-making process which requires greater understanding about patient attitudes towards treatment options.

\section{Method}

Following an initial scoping exercise, an extensive database search was undertaken and this was kept purposefully broad in order to draw on as much research as possible. Two critical appraisal tools (based on the SIGN (Scottish Intercollegiate Guidelines Network) guidelines handbook ${ }^{5}$ and the Centre for Reviews and Dissemination Guidelines $^{6}$ ) were used to help in the data extraction process.

\section{Results}

More than 500 records were identified through the search. Review of the study titles resulted in $<10 \%$ being retrieved for closer analysis, indicating a paucity of research in this area.

This presentation will discuss the results of this review, the implications for practice and future research needs.

\section{References}

1. Llewellyn CD, McGurk M, Weinman J. How satisfied are head and neck cancer (HNC) patients with the information they receive pre-treatment? Results from the satisfaction with cancer information profile (SCIP) Oral Oncology 2006; 42(7):726-734.

2. Leydon GM, Boulton M, Moynihan C, Jones A, Mossman J, Boudioni M, McPherson K. Cancer patients' information needs and information seeking behaviour: in depth interview study BMJ 1-4-2000; 320(7239): 909-913.

3. Metz JM, Devine P, DeNittis A, Jones H, Hampshire M, Goldwein J, Whittington R. A multi-institutional study of Internet utilization by radiation oncology patients. Int J Radiat Oncol Biol Phys 2003; 56(4):1201-1205.

4. Slevin M-L, Stubbs L, Plant H-J, Wilson P, Gregory W-M, Armes P-J, Downer S-M. Attitudes to chemotherapy: comparing views of patients with cancer with those of doctors, nurses, and general public. BMJ 1990; 300(6737): $1458-1460$

5. Scottish Intercollegiate Guidelines Network SIGN 50: A guideline developers handbook. http://www.sign.ac.uk/ index.html, 2004.

6. Centre for Reviews and Dissemination, York University Stage II conducting the Review: Phase 5 Study Quality Assessment CRD report 4, 2007. 